\title{
Computerized alcohol screening identified more at-risk drinkers in a level 2 than a level 1 trauma center
}

Ghasem Imani ${ }^{1,2}$, Cristobal Barrios², Craig L. Anderson ${ }^{1}$, Maryam Hosseini Farahabadi ${ }^{1}$, Faried Banimahd ${ }^{1}$, Bharath Chakravarthy ${ }^{1}$, Wirachin Hoonpongsimanont ${ }^{1}$, Christopher E. McCoy ${ }^{1}$, Georginne Mercado $^{3}$, Babak Farivar ${ }^{1}$, Jacqueline K. Pham² and Shahram Lotfipour ${ }^{1 *}$

\begin{abstract}
Background: Alcohol abuse is recognized as a significant contributor to injury. It is therefore essential that trauma centers implement screening and brief intervention (SBI) to identify patients who are problem drinkers. Although, the utility of SBI in identifying at-risk drinkers have been widely studied in level 1 trauma centers, few studies have been done in level 2 centers. This study evaluates the usefulness of SBI in identifying at-risk drinkers and to investigate the pattern of alcohol drinking among level 2 trauma patients.
\end{abstract}

Methods: This is a retrospective study of a convenience sample of trauma patients participating in computerized alcohol screening, brief intervention, and referral to treatment (CASI) in an academic level 1 trauma center and a nearby suburban community hospital level 2 trauma center. CASI utilized Alcohol Use Disorders Identification Test (AUDIT) to screen patients. We compared the pattern of alcohol drinking, demographic factors, and readiness-tochange scores between those screened in a level 2 and 1 trauma center.

Results: A total of 3,850 and 1,933 admitted trauma patients were screened in level 1 and 2 trauma centers respectively. There was no difference in mean age, gender, and language between the two centers. Of those screened, $10.2 \%$ of the level 1 and $14.4 \%$ of the level 2 trauma patients scored at-risk (AUDIT $8-19)(p<0.005$ ). Overall, 3.7\% of the level 1 and $7.2 \%$ of the level 2 trauma patients had an AUDIT score consistent with dependency (AUDIT > =20) ( $p<0.005)$. After adjusting for age, sex, education, and language, the odds of being a drinker at the level 2 center was two times of those at the level 1 center $(p<0.005)$. The odds of being an at-risk or dependent drinker at level 2 trauma center were 1.72 times of those at the level 1 center $(p<0.005)$.

Conclusions: Findings suggest that SBI is effective in identifying at-risk drinkers in level 2 trauma center. SBI was able to identify all drinkers, including at-risk and dependent drinkers at higher rates in level 2 versus level 1 trauma centers. Further studies to evaluate the effectiveness of SBI in altering drinking patterns among level 2 trauma patients are warranted.

Keywords: Level 1 trauma center, Level 2 trauma center, Computerized alcohol screening, At-risk drinking

\footnotetext{
*Correspondence: shl@uci.edu

${ }^{1}$ Department of Emergency Medicine, University of California Irvine, Orange,

CA, USA

Full list of author information is available at the end of the article
} 


\section{Background}

Excessive alcohol consumption is a risk factor for many health and societal problems. It accounts for the third leading cause of death in the United States, which is in fact preventable [1]. According to the Centers for Disease Control and Prevention (CDC), almost 30 people die each day in motor vehicle crashes that involve an alcohol-impaired driver in the United States. This is almost one death every $51 \mathrm{~min}$ [2]. In 2013, 10,076 people were killed in alcohol-related driving accidents, which accounts for $31 \%$ of all annual traffic-related deaths in the US [3]. Almost $\$ 59$ billion is spent annually on alcohol-related crashes [4].

According to the CDC, the Community Preventive Services Task Force has suggested multiple programs to reduce harmful alcohol use [5]. These measures include, the regulation of alcohol outlet density, increasing alcohol taxes, dram shop liability, maintaining limits on days of sale, maintaining limits on hours of sale, enhanced enforcement of laws prohibiting sales to minors, and electronic screening and brief intervention (e-SBI) [5]. Screening and behavioral counseling intervention is recommended by the United States Preventive Services Task Force (USPSTF) to be done in primary care in order to target individuals whose drinking patterns does not fall into alcohol dependence range but place them at risk of alcohol-related harms [6].

According to the previous data, a quarter of the US population is at-risk drinker [7].

Additionally, the prevalence of alcohol, tobacco, and other drug (ATOD) use problems among emergency department (ED) patients is $50-100 \%$ higher than the U.S. average $[8,9]$. A large percentage of ED patients have unrecognized ATOD evaluation needs and are more likely to be frequently admitted to the hospital or repeatedly utilize ED services $[9,10]$. Prior research has shown that at least $25 \%$ of all adult ED patients screen positive for hazardous or harmful drinking pattern [11, 12]. Alcohol use problems are endemic among injured trauma patients as well $[13,14]$. Thus, the widespread integration of alcohol screening and brief intervention in ED and trauma centers not only can identify those at risk, but could also be a powerful preventive tool, which has a long-standing public health impact $[15,16]$.

Multiple instruments are widely used to screen for alcohol drinking problems such as the Alcohol Use Disorders Identification test (AUDIT), AUDIT-C [17], and the Alcohol Smoking and Substance Involvement Screening Test (ASSIST) [18]. Each of these questionnaires can be utilized as a computerized version. The computerized alcohol screening, brief intervention, and referral to treatment (CASI) has been introduced by the University of California, Irvine in 2006 and has been used to screen at-risk drinkers at the ED since then. The utility of this program in screening at-risk drinkers has been widely studied in different settings [19-22].

Based on the National Trauma Data Bank 2015, there are 237 level 1 and 259 level 2 trauma centers in the US where the trauma patients receive care [23]. Alcohol SBI has been mandated by the American College of Surgeons to be done in all level 1 trauma centers since 2006 [24]. While many of these trauma patients may have alcohol and drug problems, SBI for alcohol is not mandatory in level 2 trauma centers, but only recommended. As a result, most of the studies have evaluated the effectiveness of SBI done in level 1 trauma centers while very little documentation exists regarding its use at level 2 trauma centers [25]. That means a great proportion of trauma patients who are seen at level 2 trauma centers are not offered SBI.

In this study, we hypothesize that in a level 2 community trauma center, SBI will perform its task of alcohol use disorder detection consistent with results seen at level 1 trauma centers. For this purpose, we compared the pattern of alcohol drinking, demographic factors and readiness-to-change scores between those screened in a level 2 and a level 1 trauma center using computerized SBI. If it proves to be effective in identifying alcohol use disorder in level 2 trauma centers, further studies to evaluate the usefulness of brief intervention in altering drinking patterns among these patients would be warranted and screening and brief intervention (SBI) could become standard of care in all level 2 trauma centers as well.

\section{Methods}

\section{Study design, study setting and population}

This is a retrospective study of a convenience sample of trauma patients participating in computerized SBI in a level 1 comparing to a level 2 trauma center in Orange County, California. The University of California, Irvine Human Subjects Research Institutional Review Board reviewed and approved the study protocol and waived the need for consent (HS\#2014-1330). We collected data from a level 1 university hospital and a nearby suburban community hospital level 2 Trauma center in Southern California from 2010 to 2014 inclusive.

While the trained research personnel implemented computerized SBI using a touchscreen tablet computer on all designated trauma patients admitted to the hospital in the level 1 trauma center, nurses were responsible to perform this screening in the level 2 trauma center. Patients were screened seven days a week, without interfering with patient care. All adult (age 18 and above) designated trauma patients were eligible for SBI. The computerized SBI module consists of a bilingual (English and Spanish) audio-graphical interface software program that was up-loaded onto a mobile tablet 
computer and administered at the bedside of stable trauma patients in the inpatient trauma units. It uses dynamic text, touch screen technology, and offers a text-to speech option. Headphones with Bluetooth technology are also available for patient privacy. It also recorded patients' responses and the length of time with the module. A personalized alcohol-reduction plan, along with counseling referral information, was wirelessly printed on a department printer [20, 21].

The computerized interview began with the first three questions of the Alcohol Use Disorders Identification Test (AUDIT) [26] plus an additional question about the highest number of drinks in the last month. The possible responses included the AUDIT categories for less frequent drinking, but the program inquired about the exact number of drinking days per week and the number of drinks per drinking day. The responses were used to calculate drinks per week and to assess the points for the first two AUDIT questions. The computerized SBI determined whether the patient drank more than the National Institute on Alcohol Abuse and Alcoholism (NIAAA) recommended thresholds, defined as no more than four drinks in a day and no more than fourteen drinks in a week for men age 64 years and younger and no more than three drinks in a day and no more than seven drinks in a week for women and for men age 65 years and older [27]. Patients who reported exceeding NIAAA limits were presented with the remaining AUDIT questions and the results of the screening were included in this study. Then, they are asked about their readinessto-change their alcohol drinking habit.

\section{Variables}

Trauma centers: According to the American Trauma Society, "A Level 1 Trauma Center is capable of providing total care for every aspect of injury - from prevention through rehabilitation. Elements of Level 1 Trauma Centers Include: $24-\mathrm{h}$ in-house coverage by general surgeons, and prompt availability of care in specialties such as orthopedic surgery, neurosurgery, anesthesiology, emergency medicine, radiology, internal medicine, plastic surgery, oral and maxillofacial, pediatric and critical care" [28].

"A Level 2 Trauma Center is able to initiate definitive care for all injured patients.

Elements of Level 2 Trauma Centers Include: 24-h immediate coverage by general surgeons, as well as coverage by the specialties of orthopedic surgery, neurosurgery, anesthesiology, emergency medicine, radiology and critical care, tertiary care needs such as cardiac surgery, hemodialysis and micro vascular surgery may be referred to a Level 1 Trauma Center [28].

Demographic data: Age is defined in years. It is also categorized into 6 different groups (18-20, 21-29, 30-39,
$40-49,50-64,65+)$. Sex is defined as male or female. Language is reported in two categories: English and Spanish. This is the preferred language in which patients took the test. Education was categorized into four groups: Less than high school, high school graduate, some college/ associate degree, and 4 years/advanced degree. "Some college/associate degree" serves as our reference group.

AUDIT score: This score ranges from 0-40. A score of 1-7 is categorized as low-risk although all of these patients in this sample exceeded the NIAAA recommendation levels. Scores 8-19 identifies individuals who are considered as potentially at risk for alcohol-related injuries and side effects. Pattern of alcohol dependency is defined with scores 20 and higher [26].

Readiness- to-change score (RTC): This score is a patient self-reported score using a 1-10 Likert scale [29]. Patients would be asked for their stage of change only if they report drinking above the NIAAA drinking limit. We then categorized RTC into three levels of readiness to change, minimal (scores 1 to 3 ), moderate (scores 4 to 7 ) and high (scores 8 to 10), corresponding to peaks in RTC at 1,5 , and 10 [29].

\section{Data analysis}

AUDIT Screening results and RTC scores as well as age, sex, education, language of interview were recorded by the SBI program in a comma-separated file, imported into Stata (version 14.0, StataCorp, College Station, TX), compared and then analyzed. The values were grouped into categories, which allowed the analysis to identify and present nonlinear trends without complex nonlinear models. We used the chi-square test for independence to compare demographic variables between the two trauma centers. Logistic regression was used for multivariate modeling of these associations.

\section{Results}

\section{Patient enrollment}

Overall, 4,282 and 1,998 admitted trauma patients at the level 1 and 2 trauma centers were screened using the computerized SBI during the day until midnight. We were not able to approach all eligible trauma patients due to the unavailability of research personnel over night. Among these patients, $38(0.9 \%)$ patients at the level 1 and $11(0.5 \%)$ at the level 2 refused to participate in the study. Among those agreed to participate, $394(9.2 \%)$ patients at the level1 and $54(2.7 \%)$ at the level 2 trauma centers did not complete the survey. A total of 5,783 admitted trauma patients from 2010 through 2014 were enrolled in the study. Among these, 3,850 were enrolled in the level 1 and 1,933 in the level 2 trauma center. 


\section{Demographics}

Patients' demographic characteristics are shown in the Table 1. The level 2 trauma center had more patients age 40-49 and 50-64 than the level 1 center. We observed approximately twice as many males as females in both level 1 and 2 trauma centers respectively. A total of $94.6 \%$ of patients at the level 1 and $94.5 \%$ of those at the level 2 trauma centers answered the education question. Results showed that patients at the level 2 trauma center reported higher education level; more had some college or an associate, 4-years, or advanced degree. Nearly $94 \%$ of those screened at the both trauma centers chose to take the test in English, the rest of the patients took it in Spanish.

We found a higher non-drinkers rate among level 1 trauma center patients (38.7\%) than level 2 (24.1\%), more drinkers who exceed the NIAAA thresholds in the level 2 center (25.6\% in level 1 and 32.9\% in level 2), and more at-risk and dependent drinkers among level 2 center patients. More level 1 trauma center patients identified themselves to be moderately ready to change their drinking habits; however, more level 2 patients were strongly ready to change. The same differences in readiness to change were present when logistic regression was used to control for the other variables in Table 1.

\section{Demographic factors associated with being a drinker}

Table 2 is an adjusted regression model showing the associated factors with being a drinker. After adjusting for sex, age, language, and education level, the odds of being a drinker of any amount in level 2 trauma center were two times those at the level 1 (odds ratio: $2.00,95 \% \mathrm{CI}$ $1.75-2.29)$.

Table 1 Baseline demographic characteristics

\begin{tabular}{|c|c|c|c|c|}
\hline Variables $(\mathrm{N}$ total $=5783$ ) & N/Total (\%) & Level 1 trauma center (\%) & Level 2 trauma center (\%) & $P$-value \\
\hline \multicolumn{5}{|l|}{ Age } \\
\hline $18-20$ & $517(8.9)$ & $346(9.0)$ & $171(8.8)$ & \multirow[t]{6}{*}{0.001} \\
\hline $21-29$ & $1279(22.1)$ & $846(22.0)$ & $433(22.4)$ & \\
\hline $30-39$ & $755(13.1)$ & $530(13.8)$ & 225(11.6) & \\
\hline $40-49$ & $848(14.7)$ & $529(13.7)$ & $319(16.5)$ & \\
\hline $50-64$ & $1225(21.2)$ & $787(20.4)$ & $438(22.7)$ & \\
\hline $65+$ & 1159(20.0) & $812(21.1)$ & $347(18.0)$ & \\
\hline \multicolumn{5}{|l|}{ Sex } \\
\hline Male & $3913(67.7)$ & $2602(67.6)$ & $1311(67.8)$ & \multirow[t]{2}{*}{0.85} \\
\hline Female & 1870(32.3) & $1248(32.4)$ & $622(32.2)$ & \\
\hline \multicolumn{5}{|l|}{ Education } \\
\hline Less than high school & $511(8.8)$ & $392(10.2)$ & $119(6.2)$ & \multirow[t]{4}{*}{$<.001$} \\
\hline High school graduate & $1876(32.5)$ & 1330(34.6) & $546(28.2)$ & \\
\hline Some college/Associate degree & 1835(31.7) & $1200(31.2)$ & 635(32.8) & \\
\hline 4 year/advanced degree & $1318(22.8)$ & $791(20.4)$ & $527(27.3)$ & \\
\hline \multicolumn{5}{|l|}{ Language } \\
\hline Spanish & $362(6.3)$ & $244(6.3)$ & $118(6.1)$ & \multirow[t]{2}{*}{0.73} \\
\hline English & $5421(93.7)$ & $3606(93.7)$ & 1815(93.9) & \\
\hline \multicolumn{5}{|l|}{ Drinking level } \\
\hline Non drinker & 1955(33.8) & 1489(38.7) & $466(24.1)$ & \multirow[t]{2}{*}{$<.001$} \\
\hline Within NIAAA limit & $2207(38.2)$ & 1375(35.7) & $832(43.0)$ & \\
\hline \multicolumn{5}{|l|}{ Above NIAAA limit and: } \\
\hline Low risk (AUDIT 1-7) & $668(11.5)$ & $450(11.7)$ & 218(11.3) & \\
\hline At-risk (AUDIT 8-19) & $672(11.6)$ & $394(10.2)$ & $278(14.4)$ & \\
\hline Dependent (AUDIT 20-40) & 281(4.9) & $142(3.7)$ & 139(7.2) & \\
\hline \multicolumn{5}{|l|}{ Readiness-to-change score } \\
\hline $1-3$ & $257(17.0)$ & 153(16.4) & 104(18.0) & \multirow[t]{3}{*}{0.021} \\
\hline $4-7$ & $520(34.5)$ & $346(37.2)$ & 174(30.2) & \\
\hline $8-10$ & $731(48.5)$ & $432(46.4)$ & 299(51.8) & \\
\hline
\end{tabular}


Table 2 Adjusted model for association between demographic factors and being a drinker (any amount)

\begin{tabular}{|c|c|c|c|c|}
\hline \multirow{2}{*}{$\begin{array}{l}\text { Drinker } \\
\text { Trauma center }\end{array}$} & \multirow[t]{2}{*}{ Odds ratio } & \multirow[t]{2}{*}{$P$ value } & \multicolumn{2}{|c|}{$95 \% \mathrm{Cl}$} \\
\hline & & & & \\
\hline Level $1^{\mathrm{a}}$ & Ref & Ref & Ref & \\
\hline Level 2 & 2.00 & $<0.001$ & 1.75 & 2.29 \\
\hline \multicolumn{5}{|l|}{ Age } \\
\hline $18-20$ & 0.48 & $<0.001$ & 0.38 & 0.61 \\
\hline $21-29^{\mathrm{a}}$ & Ref & Ref & Ref & \\
\hline $30-39$ & 0.82 & 0.091 & 0.65 & 1.03 \\
\hline $40-49$ & 0.55 & $<0.001$ & 0.44 & 0.68 \\
\hline $50-64$ & 0.34 & $<0.001$ & 0.28 & 0.41 \\
\hline $65+$ & 0.18 & $<0.001$ & 0.15 & 0.22 \\
\hline \multicolumn{5}{|l|}{ Sex } \\
\hline Male $^{a}$ & Ref & Ref & Ref & \\
\hline Female & 0.50 & $<0.001$ & 0.44 & 0.57 \\
\hline \multicolumn{5}{|l|}{ Education level } \\
\hline Less than high school & 0.80 & 0.055 & 0.64 & 1.00 \\
\hline High school graduate ${ }^{a}$ & Ref & Ref & Ref & \\
\hline Some college/Associate degree & 1.32 & $<0.001$ & 1.13 & 1.53 \\
\hline 4 year/advanced degree & 1.30 & 0.002 & 1.10 & 1.54 \\
\hline \multicolumn{5}{|l|}{ Language } \\
\hline English $^{\mathrm{a}}$ & Ref & Ref & Ref & \\
\hline Spanish & 0.79 & 0.073 & 0.61 & 1.02 \\
\hline Constant & 4.04 & $<0.001$ & 3.40 & 4.80 \\
\hline
\end{tabular}

Reference group for comparison (pseudo $\mathrm{R}^{2}=0.11$ )

\section{Demographic factors associated with being at-risk or dependent drinker}

As shown in the Table 3, after controlling for age, sex, language, and education level, the odds of being an atrisk or dependent drinker (AUDIT $>=8$ ) at level 2 were 1.72 times those at the level 1 trauma center (odds ratio $1.72,95 \% \mathrm{C} 1$ 1.48-2.00).

\section{Discussion}

To our knowledge, this is one of the few studies done at a level 2 trauma center to examine the utility of SBI and to evaluate its usefulness in finding at-risk drinkers among the trauma patients seen at a level 2 trauma center [25]. Our findings suggest that SBI is successful in identifying at-risk and dependent alcohol drinkers in a level 2 trauma center. While the results showed that almost $13.9 \%$ of individuals screened in the level 1 trauma center were identified by AUDIT as at-risk or dependent, this number was $21.6 \%$ in the level 2 trauma center. The results also showed that there are more drinkers among level 2 trauma patients in all age groups compared to the level 1. It should not be overlooked that the population screened at the level 2 trauma center had higher
Table 3 Adjusted model for association between demographic factors and showing the patterns of at-risk drinking or dependency (AUDIT > =8)

\begin{tabular}{|c|c|c|c|c|}
\hline \multirow{2}{*}{$\begin{array}{l}\text { At-risk drinking or dependency } \\
\text { Trauma center }\end{array}$} & \multirow[t]{2}{*}{ Odds ratio } & \multirow[t]{2}{*}{$P$ value } & \multicolumn{2}{|c|}{$95 \% \mathrm{Cl}$} \\
\hline & & & & \\
\hline Level $1^{\mathrm{a}}$ & Ref & Ref & Ref & \\
\hline Level 2 & 1.72 & $<0.001$ & 1.48 & 2.007 \\
\hline \multicolumn{5}{|l|}{ Age } \\
\hline $18-20$ & 0.63 & 0.001 & 0.48 & 0.82 \\
\hline $21-29^{a}$ & Ref & Ref & Ref & \\
\hline $30-39$ & 0.93 & 0.58 & 0.74 & 1.17 \\
\hline $40-49$ & 0.70 & 0.003 & 0.56 & 0.88 \\
\hline $50-64$ & 0.53 & $<0.001$ & 0.42 & 0.66 \\
\hline $65+$ & 0.23 & $<0.001$ & 0.17 & 0.31 \\
\hline \multicolumn{5}{|l|}{ Sex } \\
\hline Male ${ }^{a}$ & Ref & Ref & Ref & \\
\hline Female & 0.40 & $<0.001$ & 0.33 & 0.49 \\
\hline \multicolumn{5}{|l|}{ Education level } \\
\hline Less than high school & 0.91 & 0.519 & 0.69 & 1.20 \\
\hline High school graduate ${ }^{a}$ & Ref & Ref & Ref & \\
\hline Some college/Associate degree & 0.72 & $<0.001$ & 0.60 & 0.86 \\
\hline 4 year/advanced degree & 0.62 & $<0.001$ & 0.49 & 0.77 \\
\hline \multicolumn{5}{|l|}{ Language } \\
\hline English $^{a}$ & Ref & Ref & Ref & \\
\hline Spanish & 0.79 & 0.16 & 0.58 & 1.09 \\
\hline Constant & 0.39 & $<0.001$ & 0.32 & 0.46 \\
\hline
\end{tabular}

education level. The effect of education on alcohol drinking habits is controversial. While some studies proved no association between level of education and alcohol drinking [30,31], others showed that lower education level is associated with higher level of drinking [32]. Overall, the effect of demographic factors was not strong enough to explain the identification of higher number of drinkers found at the level 2 in our study. The odds of being a drinker of any amount is still 2.00 times in patients screened in the level 2 than the level 1 trauma center. Even assuming the same demographic factors, the likelihood of individuals being an at-risk or dependent drinker is 1.72 times in level 2 compared to those screened in level 1. Additionally, our data showed that a greater number of patients in level 2 trauma center exhibited strong willingness to change their alcohol drinking habits compared to the level 1 .

For every individual in the US who is alcohol dependent, there are six other adults who are at risk for injury due to their alcohol drinking habits. As a matter of fact, this population is more likely to utilize trauma center services [33]. It has been reported that $30 \%-50 \%$ of trauma center 
cases are alcohol related [34]. SBI in primary care can result in a $15-30 \%$ reduction in alcohol consumption lasting for at least a year [35, 36]. Screening and brief intervention provided in EDs is more effective at a lower cost compared to screening provided to the outpatients [37] and proved to be effective in reducing hazardous alcohol drinking behavior at 6 months follow-up in non-dependent group screened at level 1 trauma and ED [38]. The reasons for screening level 1 trauma patients apply equally to level 2 trauma patients. In the two centers we examined, levels of alcohol use were greater at the level 2 trauma center with a greater number of patients demonstrating high level of readiness-to-change. Given the ease of computerized screening, failure to screen level 2 patients is a missed opportunity to reduce subsequent injuries among trauma patients.

Nonetheless, studies have shown that ED directors might not be familiar with the SBI or might be unaware of it being mandatory [35]. There are barriers such as time constraint and financial resources that causes the SBI to lag behind the national guidelines [39]. Alcohol related problems are the health issues that are mostly addressed by computerized health interventions [40]. Computerized screening performs better in finding atrisk drinkers in all age groups compared to medical screening examination [22]. This may be due to patient's' preference to disclose their sensitive health information to computers compared to health care staff and they are more likely to share honest answers [41]. It further improves patients' knowledge of safe drinking and delivers alcohol education in a comfortable way [42].

Our study proved that SBI is as useful in finding atrisk and dependent drinkers at a level 2 trauma center as a level 1 trauma center. Therefore, we suggest further studies to evaluate the barriers to the mandatory implementation of SBI in all level 2 trauma centers.

\section{Limitation}

This study uses convenience samples at only two institutions. Patients were not enrolled overnight or when the emergency department and trauma service experienced heavy patient volumes. The fact that we could not control for other socio economic status such as income, employment, ethnicity etc. limited our ability to analyze the differences between patients of the two centers. The focus of our study was on the feasibility of SBI in level 2 trauma center and to evaluate its usefulness in identifying at-risk drinkers. Thus, the next step in studying SBI would be to investigate the effectiveness of intervention in altering alcohol drinking behavior.

The personnel conducting the computerized model of SBI were different at the two institutions. Although the differences between institutions prevent a direct comparison, nurses may be more effective in enrolling patients and encouraging completion. This is likely due to their familiarity with the patient or being able to time the intervention to the patient's openness to it. This may account for some of the differences we found between the two institutions.

\section{Conclusions}

We identified that SBI performed in level 2 trauma centers was as effective in level 1 trauma centers in revealing at-risk drinkers. This population of patients was recognized as being in a vulnerable state due to the cause and event bringing them to a trauma center. Continuing to target this population with SBI and provide outreach and awareness is markedly essential in both level 1 and 2 trauma centers.

\section{Abbreviations}

ASSIST: Alcohol smoking and substance involvement screening test; ATOD: Alcohol, tobacco, and other drug; AUDIT: Alcohol use disorders identification test; CASI: Computerized alcohol Screening brief Intervention, and referral to treatment; CDC: Centers for disease control and prevention; ED: Emergency department; e-SBI: Electronic screening and brief intervention; SBI: Screening and brief intervention; USPSTF: United States preventive services task force

\section{Acknowledgements}

Most importantly the authors thank Marcia Noakes and Daniel Bondaranko for their great contribution to the project. Thank you to the Emergency Medicine Research Associates Program for their assistance with CASI. We appreciate the assistance of Ronald Sahyouni and Brianna Marie Miner.

\section{Funding}

No funding was provided for this study.

\section{Availability of data and material}

Data is fully available upon request through the corresponding author.

\section{Authors' contributions}

$C A, S L, C B, G I, M H$, and GM carried out the study design, the acquisition of data and data analysis. $G M, C M, B C, J P, B F, W H$, and FB were involved in interpretation of the data as well as drafting the manuscript. $C A, C B, M H, G l$, and SL drafted the final version of the manuscript and performed revisions including the critical revisions in response to the reviewers' comments. All authors have read and agreed to the final version of the manuscript and will be accountable for all aspects of the work.

\section{Competing interests}

The authors declare that they have no competing interests.

\section{Consent for publication}

Not applicable.

\section{Ethics approval and consent to participate}

Since no public health identifier was collected at the time of data acquisition and analysis for this study, the need to obtain informed consent was deemed unnecessary. Thus, the University of California, Irvine Human Subjects Research Institutional Review Board reviewed and approved the study protocol and waived the need for consent (HS\# 2014-1330).

\section{Author details}

'Department of Emergency Medicine, University of California Irvine, Orange, CA, USA. ${ }^{2}$ The Division of Trauma, Burns, Critical Care and Acute Care

Surgery, University of California Irvine, Orange, CA, USA. ${ }^{3}$ Trauma Services at St. Joseph Health, Mission Viejo, CA, USA.

Received: 11 July 2016 Accepted: 22 December 2016

Published online: 06 January 2017 


\section{References}

1. Bouchery EE, Harwood HJ, Sacks JJ, et al. Economic costs of excessive alcohol consumption in the U.S. 2006. Am J Prev Med. 2011;41(5):516-24.

2. Department of Transportation (US), National Highway Traffic Safety Administration (NHTSA). Traffic Safety Facts 2013 Data: Alcohol-Impaired Driving.2014. http://www-nrd.nhtsa.dot.gov/Pubs/812102.pdf. Accessed 10 Feb 2016

3. Department of Justice (US), Federal Bureau of Investigation (FBI). Crime in the United States 2010: Uniform Crime Reports. Washington (DC): FBI 2011 http://www.fbi.gov/about-us/cjis/ucr/crime-in-the-u.s/2010/crime-in-the-u.s.2010/summary. Accessed 10 Feb 2016.

4. Blincoe L, Miller TA, Zaloshnja E, Lawrence BA. The Economic and sociatal Impact of Motor Vehicle Crashes. Dept of Transportation (US), National Highway Traffic Safety Administration (NHTSA). 2010-revised. https:// crashstats.nhtsa.dot.gov/Api/Public/ViewPublication/812013.

5. Centers for Disease Control and Prevention. Fact Sheets - Preventing Excessive Alcohol Use. 2015. http://www.cdc.gov/alcohol/fact-sheets/ prevention.htm. Accessed 8 Jan 2016

6. US Preventive Services Task Force. Screening and Behavioral Counseling Interventions in Primary Care to Reduce Alcohol Misuse: Recommendation Statement. 2013. https://www.guideline.gov/summaries/summary/45519.

7. Saitz R. Alcohol screening and brief intervention in primary care: absence of evidence for efficacy in people with dependence or very heavy drinking. Drug Alcohol Rev. 2010;29(6):631-40.

8. Cherpitel CJ, Ye Y, Bond J. Attributable risk of injury associated with alcohol use: cross national data from the emergency room collaborative alcohol analysis project. Am J Public Health. 2005;95(2):266-72.

9. Hankin A, Daugherty M, Bethea A, Haley L. The Emergency Department as a preventionsite: a demographic analysis of substance use among ED patients. Drug Alcohol Depend. 2013;130(1-3):230-3.

10. Rockett IR, Putnam SL, Jia H, Chang CF, Smith GS. Unmet substance abuse treatmentneed, health services utilization, and cost: a population-based emergency department study. Ann Emerg Med. 2005;45(2):118-27.

11. Aseltine Jr RH, Schilling EA, James A, Murray M, Jacobs DG. An evaluation of National Alcohol Screening Day. Alcohol Alcohol. 2008;43(1):97-103.

12. Academic ED. SBIRT Research Collaborative. The impact of screening, brief interventionand referral for treatment in emergency department patients' alcohol use. Ann EmergMed. 2007:50(6):699-710.

13. Soderstrom CA, Smith GS, Dischinger PC, McDuff DR, Hebel JR, Gorelick DA, et al. Psychoactive substance use disorders among seriously injured trauma center patients. JAMA. 1997;277(22):1769-74.

14. Zatzick D, Donovan D, Dunn C, Russo J, Wang J, Jurkovich G, et al. Substance use and PTSD in trauma center patients receiving mandated alcohol SBI. J Subst Abuse Treat. 2012;43(4):410-7.

15. Koepsell TD, Zatzick DF, Rivara FP. Estimating the population impact of preventive interventions from randomized trials. Am J Prev Med. 2011;40(2):191-8

16. Jonas DE, Garbutt JC, Amick HR, Brown JM, Brownley KA, Council CL, et al. Behavioral counseling after screening for alcohol misuse in primary care: a systematic review and meta-analysis for the U.S. preventive services task force. Ann Intern Med. 2012;157(9):645-54

17. Bush K, Kivlahan DR, McDonell MB, Fihn SD, Bradley KA. The AUDIT alcohol consumption questions (AUDIT-C): an effective brief screening test for problem drinking. Ambulatory Care Quality Improvement Project (ACQUIP). Alcohol Use Disorders Identification Test. Arch Intern Med. 1998;158(16):1789-95.

18. Alcohol, Smoking and Substance Involvement Screening Test (ASSIST). World Health Organization (WHO). 2010. http://apps.who.int/iris/bitstream/ 10665/44320/1/9789241599382_eng.pdf. Accessed 5 June 2016.

19. Vaca FE, Winn D, Anderson CL, Kim D, Arcila M. Six-month follow-up of computerized alcohol screening, brief intervention, and referral to treatment in the emergency department. Subst Abus. 2011:32(3):144-52.

20. Lotfipour S, Cisneros V, Chakravarthy B, Barrios C, Anderson CL, Fox JC, et al. Assessment of Readiness-to-Change and Relationship to AUDIT Score in a Trauma Population Utilizing Computerized Alcohol Screening and Brief Intervention. Subst Abus. 2012;33(4):378-86.

21. Vaca F, Winn D, Anderson C, Kim D, Arcila M. Feasibility of emergency department bilingual computerized alcohol screening, brief intervention, and referral to treatment. Subst Abus. 2010;31:264-9.

22. Lotfipour S, Howard J, Roumani S, Hoonpongsimanont W, Chakravarthy B, Anderson $\mathrm{CL}$, et al. Increased Detection of Alcohol Consumption and At-risk Drinking with Computerized Alcohol Screening. J Emerg Med. 2013;44(4):861-6.
23. National Trauma Data Bank. 2015. https://www.facs.org/ /media/files/ quality\%20programs/trauma/ntdb/ntdb\%20annual\%20report\%202015.ashx. Accessed 10 June 2016.

24. American College of Surgeons. Alcohol Screening and Brief Intervention (SBI) for Trauma Patients: Committee on Trauma Quick Guide. 2007. https:// www.facs.org/ /media/files/quality\%20programs/trauma/publications/ sbirtguide.ashx. Accessed 10 Feb 2016.

25. Reed Jr DN, Wolf B, Barber KR, Kotlowski R, Montanez M, Saxe A, et al. The stages of change questionnaire as a predictor of trauma patients most likely to decrease alcohol use. J Am Coll Surg. 2005;200(2):179-85.

26. Babor TF, Higgins-Biddle JC, Saunders JB, Monteiro MG. AUDIT: The Alcohol Use Disorders Identification Test. World Health Organization. 2001.http:// apps.who.int/iris/bitstream/10665/67205/1/WHO_MSD_MSB_01.6a.pdf

27. National Institute on Alcohol Abuse and Alcoholism. Helping Patients Who Drink Too Much: A Clinician's Guide, Washington DC. 2005. https://www. niaaa.nih.gov/guide\#guide. Accessed 10 Feb 2016.

28. American Trauma Society: Trauma center levels explained. http://www. amtrauma.org/?page=traumalevels (2015). Accessed 15 Oct 2016

29. Bernstein E, Bernstein J, Project LS, ASSERT. An ED- based intervention to increase access to primary care, preventive services, and the substance abuse treatment system. Ann Emerg Med. 1997;30(2):181-97.

30. Casswell S, Pledger M, Hooper R. Socioeconomic status and drinking patterns in young adults. Society for the study of addiction to alcohol and other drugs. Addiction. 2003;98:601-10.

31. Halme JT, Seppa K, Alho H, Sami P, Poikolainen K, Lönnqvist J, et al. Hazardous drinking: prevalence and associations in the finnish general population alcoholism: clinical and experimental research. Alcohol Clin. 2008;32:9.

32. Dias P, Oliveira A, Lopes C. Social and behavioral determinants of alcohol consumption. Ann Hum Biol. 2011;38(3):337-44.

33. Alcohol Screening and Brief Intervention for Trauma patients. https://www. facs.org/ /media/files/quality\%20programs/trauma/publications/sbirtguide. ashx. Accessed 10 June 2016

34. Trinks A, Festin K, Bendtsen P, Nilsen P. What makes emergency department patients reduce their alcohol consumption? - A computer-based intervention study in Sweden. Int Emerg Nurs. 2013;21:3-9.

35. Kaner EF, Beyer F, Dickinson HO, Pienaar E, Campbell F, Schlesinger C, et al. Effectiveness of brief alcohol interventions in primary care populations. Cochrane Database Syst Rev. 2007;2:CD004148

36. Moyer A, Finney JW, Swearingen CE, Vergun P. Brief interventions for alcohol problems: a meta-analytic review of controlled Investigations in treatment-seeking populations. Addiction. 2002;97(3):279-92.

37. Barbosa C, Cowell A, Bray J, Aldridge A. The Cost-effectiveness of Alcohol Screening, Brief Intervention, and Referral to Treatment (SBIRT) in Emergency and Outpatient Medical Settings. J Subst Abus Treat. 2015;53:1-8.

38. Sommers MS, Lyons MS, Fargo JD, Sommers BD, McDonald CC, Shope JT, Fleming MF, et al. Emergency department-based brief intervention to reduce risky driving and hazardous/harmful drinking in young adults: a randomized controlled trial. Alcohol Clin Exp Res. 2013;37(10):1753-62.

39. Cunningham RM, Harrison SR, McKay MP, Mello MJ, Sochor M, Shandro JR, et al. National survey of emergency department alcohol screening and intervention practices. Ann Emerg Med. 2010;55(6):556-62.

40. Choo EK, Ranney ML, Aggarwal N, Boudreaux ED. A systematic review of emergency department technology-based behavioral health interventions. Acad Emerg Med. 2012;19:318-28.

41. De Leeuw E, Hox J, Kef S. Computer-Assisted self interviewing tailored for special populations and topics. Field Methods. 2003;15:223-51.

42. Murphy MK, Bijur PE, Rosenbloom D, Bernstein SL, Gallagher EJ. Feasibility of a computer-assisted alcohol SBIRT program in an urban emergency department: patient and research staff perspectives. Addict Sci Clin Pract. 2013;8:2 